\title{
COGNICIÓN Y LENGUAJE EN NIÑOS CRI-DU-CHAT Y DOWN. AVANCES DE UN ESTUDIO COMPARATIVO
}

\section{COGNITION AND LANGUAGE IN CHILDREN CRI-DU-CHAT AND DOWN. A COMPARATIVE STUDY PROGRESS}

\author{
María Paz Fernández Lozano \\ Universidad Complutense de Madrid, España \\ Aníbal Puente Ferreras \\ Universidad Complutense de Madrid, España
}

\begin{abstract}
Resumen: La genética humana ofrece la posibilidad de comprender enfermedades que hasta hace poco tiempo eran desconocidas, tales como el Cri-du-Chat (CDC), X frágil y Prader-Willi entre otras. Descubrir la etiología genética de una enfermedad es importante pero no agota la información necesaria para rehabilitar a los afectados. Hasta hoy no existe un tratamiento médico restaurador para el Cri-du-Chat y el síndrome Down (SD). El propósito del trabajo es conocer el funcionamiento cognitivo y lingüístico de estas personas y analizar de qué forma estos factores contribuyen a mejorar su rendimiento académico y personal. Se trata de un estudio comparativo de los patrones de conducta observados en los SD y CDC. Para el logro del objetivo revisamos la literatura reciente tomando en cuenta la etiología, el diagnóstico, la evolución de la enfermedad, la intervención, etc. Un resultado significativo es que el conocimiento de los CDC es todavía muy escaso en comparación con el desarrollo alcanzado con el SD, particularmente en las áreas cognitivas y lingüísticas.
\end{abstract}

Palabras clave: Síndrome Down, Síndrome Cri-du-Chat, retardo mental, cognición, lenguaje, anormalidades cromosómicas, memoria, atención.

\begin{abstract}
Human Genetics offers the possibility of understanding certain diseases that were practically unknown until recently, such as Cri-du-Chat, Fragile X and Prader-Willi among others. Discovering the genetic etiology of a complex disease is important, but not a substitute for the information required for the rehabilitation of those affected. Currently there is no restorative treatment for Cri-du-Chat (CDC) and Down syndrome (DS). The purpose of this paper is to understand the cognitive and linguistic functioning of these patients, and to analyze the ways in which these factors contribute to improve their academic and personal performance. In this article, the author presents (O: THE AUTHORS PRESENT, SI FUERAN DOS O MÁS AUTORES) a comparative study of the behaviour patterns observed in DS and CDC. To achieve this objective we review recent literature taking into account the etiology, diagnosis, evolution of the disease, and intervention techniques, among other aspects. A significant outcome is the fact that the levels of knowledge about CDC syndrome are still very low compared to those achieved through research on DS, particularly in cognitive and linguistic areas.
\end{abstract}

Keywords: Down Syndrome; Cri-du-Chat Syndrome, mental retardation, cognition, language, chromosomal abnormalities, memory, attention.

\section{INTRODUCCIÓN}

Entre las enfermedades descritas en la literatura médica de origen cromosómico, que cursan con retardo mental moderado o grave, el síndrome Down es una de las más paradigmáticas. Desde hace siete décadas se viene hablando de la posibilidad de restaurar la anomalía cromosómica utilizando para ello mecanismos de intervención molecular.
A fecha de hoy, se puede afirmar que los resultados conocidos y su eficacia se pueden considerar de muy pobres y decepcionantes para la comunidad científica y sobre todo para las familias.

El Síndrome Down (SD) no es una de las "enfermedades raras", puesto que existe un importante número de personas que la padecen, el "corpus científico" de conocimientos acerca 
de cómo se produce es abundante y novedoso y es una de las enfermedades de origen genético que primero se descubrieron (año 1866) (Rondal, Perera \& Nadel, 2000).

Sin embargo, todavía no se conoce la razón última que explique la aparición de esta alteración cromosómica, de cuya existencia tenemos constancia desde 1500 años A. C. (Rogers \& Coleman, 1994).

A diferencia del Down, el síndrome Cridu-Chat (CDC) es uno de las denominadas "enfermedades raras" ya que presenta muy baja incidencia y su principal característica es el llanto producido por los bebés, similar al maullido de un gato, que viene provocado por una hipoplastia de la laringe. Un aspecto común al Down y al CDC es el retardo mental que oscila entre moderado y leve, aunque en ambos casos el déficit cognitivo puede llegar a ser severo con enormes dificultades para el desarrollo autónomo. El rápido diagnóstico de la enfermedad es importante para mitigar los efectos en el retraso intelectual.

Algo que diferencia de una manera muy marcada el Down y el CDC es que sobre este último existen muchas áreas desconocidas.

El desarrollo científico alcanzado desde su descubrimiento hasta la fecha es relativamente escaso. Se conoce la base genética de origen y unos pocos casos descriptivos a partir de los cuales hemos podido conocer el perfil físico y algunos rasgos conductuales de los pacientes. Sin embargo, otros aspectos de la enfermedad permanecen todavía en la oscuridad. Conocer las debilidades y sobre todo las fortalezas de los CDC es algo que todavía está por analizar. Particularmente es necesario determinar el funcionamiento de su aparato mental (memoria, atención, lenguaje, lectura, escritura, etc.) y su representación en el cerebro, como elementos funcionales que podrían proyectar información sobre las posibles metas personales y académicas que los pacientes pudieran alcanzar (Rondal, Perera \& Nadel, 2000).

Hace tiempo se pensaba y postulaba que el elemento clave en el retraso mental estaba en el coeficiente intelectual $(\mathrm{Cl})$ conjuntamente con una estimación del potencial de adaptación del individuo. Este punto de vista, aunque no del todo incierto, resulta sin embargo de- masiado genérico. La perspectiva científica actual ante el retraso mental debería estar más enfocada hacia la etiología. De hecho, los recientes avances en el campo de la genética han mostrado la importancia que los fenotipos de comportamiento tienen en los síndromes de retraso mental (Dykens \& Clarke, 1997). Se ha demostrado que distintos síndromes de retraso mental localizados en niveles de $\mathrm{Cl}$ similares (en valores medios para los sujetos afectados por el síndrome) difieren de forma sustancial en cuanto al número de aspectos psicológicos relacionados.

Las comparaciones del SD y Síndrome Cridu-Chat desde el enfoque etiológico nos permite determinar dónde puede originarse las semejanzas y las diferencias en aspectos cognitivos como memoria, atención y nivel intelectual y en aspectos lingüísticos como producción y comprensión del lenguaje oral y escrito. La etiología se ha convertido en una variable predictora de gran importancia en el ámbito del retraso mental. Esto se puede ver claramente en los síndromes genéticos que producen retraso mental, puesto que los defectos iniciales de estos síndromes pueden aparecer identificados a nivel cromosómico o a nivel genético. Descubrir el perfil de ventajas y desventajas puede proporcionar información de utilidad para los profesionales de la educación especial. Hoy día, por ejemplo, se conoce que la capacidad lingüística en el SD no es prototípica del retraso mental moderado o severo. Se puede deducir que las diferencias de lenguaje entre los síndromes de retraso mental provienen de diferencias a nivel cerebral que probablemente tengan su origen en la diversidad genética característica de estos síndromes.

El propósito que nos planteamos es un análisis comparativo y una revisión actualizada de los rasgos que comparten ambos síndromes y aquéllos que son específicos y diferenciados. En el desarrollo del trabajo, obviaremos los contenidos más descriptivos y conocidos (particularmente del SD) y nos concentraremos en asuntos que han sido menos explorados por la comunidad científica y que representan áreas de gran interés. Los aspectos de mayor relevancia objeto del estudio tienen que ver con el análisis de las funciones cognitivas y el aparato lingüístico. Nuestro enfoque es más prospectivo que retrospectivo. 
Preferimos enfatizar más en los puntos fuertes que en los débiles para tratar de descubrir sus potencialidades y posibilidades de desarrollo intelectual y comunicativo.

Comenzaremos con una revisión del CDC, que es un síndrome relativamente desconocido.

\section{SINDROME CDC: ETIOLOGIA}

EI CDC fue descrito por primera vez en el año 1963 por el Dr. Jerôme Lejeune en tres pacientes no emparentados (Lejeune, Lafourcade, Berger, Vialatte, Boeswillwald, Seringe et al, 1963). El síndrome es una entidad infrecuente que presenta un rango de incidencia de $1: 15.000$ a 1:50.000 en recién nacidos vivos (Cerruti Mainardi, 2006). Nieburhr (1978) encontró una incidencia de alrededor de 1:350 en 6000 personas con retardo mental y Duarte, Cunha, Roth, Ferreira, Garcías y Martino-Roth (2004) hallaron una frecuencia de 1:305 en 916 pacientes a quienes se les realizó un estudio citogenético. La enfermedad predomina en niñas, aunque afecta a ambos sexos y a todas las razas. El CDC se incluye en la categoría de anomalías estructurales cromosómicas. Este tipo se caracteriza por la rotura de un cromosoma con consecuencias desconocidas (Tsao, Wenger \& Bartholomew 2005). En el $85-90 \%$ de los casos, el síndrome se da por delección, translocación o pérdida parcial en el brazo corto del cromosoma 5, ocurrida en el mismo paciente y en el $10-15 \%$ restante lo heredan de sus padres. En esta rara supresión cromosómica es difícil hablar de causas concretas, pero se establece que en la mayoría de los casos se debe a la pérdida espontánea de una parte del cromosoma 5 durante el desarrollo de un óvulo o de un espermatozoide (Cerruti Mainardi, 2006).

\section{DIAGNÓSTICO}

El diagnóstico, generalmente, se realiza en el momento del nacimiento. Si bien, el síntoma más llamativo es el llanto persistente y característico, sólo se puede confirmar por el análisis cromosómico ((Cerruti Mainardi, Perfumo, Calí, et al, 2001; Cerruti Mainardi, Guala, Pastore, et al, 2000; Stathopulu, Mackie Ogilvie \& Flinter, 2003). El diagnóstico prenatal mediante ecografía es difícil y el CDC no tiene un tratamiento específico disponible, por lo tanto es fundamental que los padres se informen en profundidad lo antes posible respecto al síndrome y, de conocer antecedentes en la familia, lo comuniquen a sus médicos. Teniendo en cuenta esta información, deben buscar asesoramiento genético y someterse a una prueba de cariotipo con el fin de determinar si uno de ellos tiene un re-arreglo del cromosoma 5 (Cerruti Mainardi, 2003). Generalmente se encara un abordaje multidisciplinario, y en la mayoría de los casos centrado en el retraso mental.

En relación al trabajo familiar y los cuidados básicos, se recomienda que los padres presten especial atención en los siguientes aspectos: - Actuación precoz, comenzar los trabajos de estimulación temprana una vez que se haya determinado el cuadro. La familia debe colaborar y tener las orientaciones y apoyos precisos por parte de los profesionales que atienden al niño. - Asumir la necesidad de buscar soluciones y tratamientos. - Colaboración entre los profesionales, ejerciendo el papel de coordinadores, exigiendo información y coordinación del trabajo diario, con el fin de no distorsionar los aprendizajes conseguidos y participar así de los avances obtenidos y las limitaciones surgidas.

\section{CARACTERÍSTICAS}

Si bien el diagnóstico se sospecha por el llanto persistente característico, sólo se puede confirmar por el análisis puntual cromosómico (Fang, Lee, Huang, Syu, Yang, Wang et al, 2008). En todos los casos el síndrome produce minusvalía psíquica o retraso mental y el impacto de esa anomalía depende de lo que suceda con los fragmentos implicados (Carlin, 1988a). Los niños se desarrollan lentamente y permanecen muy retrasados en cuanto a su estática y psicomotricidad (Wikins, Brown \& Wolf, 1980; Cerruti Mainardi, Guala, Pastore, Pozzo, Dagna Bricarelli, \& Pierluigi, 2000; Cerruti Mainardi, Perfumo, Calì, Coucourde Pastore, Cavani, S. et al, 2001). Al aumentar la edad puede acentuarse el retraso de las capacidades intelectuales. Sin embargo, el pronóstico de los afectados está estrechamente vinculado a las malformaciones derivadas y se asocia con 
retraso psicomotor. A parte de estos rasgos característicos conviene considerar otros que a veces se muestran en algunos pacientes de manera más infrecuente. Conviene recordar que si hay algo que caracteriza el CDC es la heterogeneidad de los rasgos físicos (Colins \& Eaton-Evans, 2001), mientras que el retraso mental y psicomotor nunca fallan y además se mantienen a lo largo de su ciclo vital. Veamos un cuadro de rasgos frecuentes y menos frecuentes que puede encontrarse en las descripciones clínicas.

Entre las malformaciones cráneo-faciales más frecuentes encontramos: cráneo pequeño, posible asimetría facial y craneal, cara redonda, ojos separados, orejas con implantación baja, mandíbula pequeña, pliegue de piel en el ángulo interno del ojo, raíz nasal prominente, paladar en forma de bóveda, malocusión dental, estrabismo, miopía, astigmatismo, anomalías del iris, cuello corto, labio superior partido, manos pequeñas, arqueamiento permanente de un dedo, fusión congénita o accidental de dos o más dedos, pliegue simiesco (un solo pliegue en las palmas de las manos), pies planos o zambos. Además de las malformaciones craneales también encontramos alteraciones músculo-esqueléticas como hipotonía, luxación de cadera, laxitud ligamentosa, escoliosis a partir de los 8 años de edad, anomalías vertebrales, huesos iliacos y esternón pequeño, hernia inguinal (Niebuhr, 1979; Cammarata-Scalisi, Araque, Da Silva \& Hernández, 2009).

\section{DESARROLLO PSICOMOTOR}

Existe escasa literatura científica referida al desarrollo psicomotor de los CDC, y la poca que existe señala que estos sujetos presentan retrasos severos en el área motora y mental (Niebuhr, 1978; Breg, Steele, Miller, Warburtonb, Capoa \& Allerdice, 1970). Sin embargo, los informes de las familias de niños con CDC que han sido tratados precozmente y de forma adecuada son más optimistas. El retraso evolutivo se hace evidente desde la etapa intrauterina y posteriormente su desarrollo sigue siendo lento (Marinescu, Cerruti Mainardi, Collins, Kouahou, Coucourde, Pastore, Eaton-Evans \& Overhauser, 2000). La motricidad, el equilibrio y las capacidades intelectuales presentan un retraso significativo a medida que los niños crecen (Breg,
Steele, Miller, Warburtonb, Capoa \& Allerdice, 1970; Niebuhr, 1978). De los 2 a los 5/6 años, los pacientes suelen mantener hipotonicidad general lo que determina que sean sumamente frágiles a la hora de andar, mantener el equilibrio o efectuar cualquier actividad que tenga implicaciones motoras. La boca suele estar abierta y la mandíbula, relativamente pequeña, suele producir mala oclusión dental lo que dificulta el reflejo de morder y engullir la comida.

Los neonatos frecuentemente presentan serios problemas de asfixia, crisis cianóticas e impedimentos para succionar. Este último inconveniente agrava el cuadro ya que estos niños suelen mostrar bajo peso al nacer. A lo largo del primer año aparecen otros problemas con particular virulencia que afectan el desarrollo motor, aunque no sean excesivamente frecuentes: alteraciones cardiacas, neurológicas, renales y malformaciones en la base craneal (Niebuhr, 1978). En relación con el maullido se conoce que su típica expresión tiene que ver con anormalidades en la laringe (pequeña, estrecha y en forma de diamante) y en la epiglotis (pequeña, hipotónica y flácida) (Rizzi, 1997). A lo largo del desarrollo suelen presentarse otros problemas derivados de las limitaciones motoras como son: problemas respiratorios, de oído y también de la vista que necesitarán tratamiento por parte de los médicos especialistas (Choong, Watts, Little \& Beck, 2003; Kitsiou-Tzeli, Dellagrammaticas, Papas, Ladas \& Bartsocas, 1983).

Uno de los estudios más significativos relacionado con el desarrollo psicomotor fue el llevado a cabo por Wilkins, Brown, Nance y Wolf (1982). Los datos obtenidos con 86 niños con CDC evidencian una EM de alrededor de 2 años y 6 meses con una EC de 6 y 7 años. La EM y los cocientes de desarrollo social son inversamente proporcionales a la EC en las cuales se ha iniciado la intervención precoz. Carlin (1988b) ofrece datos longitudinales sobre 31 individuos con CDC y datos transversales sobre otros 31 casos. En todos los individuos con CDC, se observa un retraso del crecimiento notable, microcefalia, un importante retraso psicomotor e infecciones respiratorias y óticas frecuentes. También encuentra hipotonía generalizada durante los primeros meses que posteriormente tiende a normalizarse en al menos el $50 \%$ de los casos si viene acompañada de entrenamiento precoz. 
Las metas evolutivas que los afectados puedan alcanzar vendrán condicionadas por tres factores:

1. La magnitud de los daños estructurales en el brazo corto del Cromosoma 5 y en las zonas concretas afectadas. La magnitud del daño determinará el límite biológico y la posibilidad de alcanzar metas específicas de tipo físico, cognitivo, social y personal (Cerruti Mainadi, 2006).

2. La atención precoz suministrada así como el entrenamiento y/o enseñanza recibida por parte de profesionales especializados.

3. La respuesta del niño. Generalmente se asume que cuando el niño muestra interés por la interacción, curiosidad por el entorno y deseo de aprender, éstos son signos que hacen prever un mejor pronóstico y evolución (Choong, Watts, Little \& Beck, 2003).

\section{EVOLUCIÓN COGNITIVA Y LINGÜÍSTICA}

Si el conocimiento del desarrollo psicomotor de los CDC es muy limitado, en lo referido al desarrollo del lenguaje y la cognición todavía es más reducido. En este apartado vamos a revisar algunos de los trabajos de investigación más significativos comenzando con el lenguaje. Las observaciones y los registros confirman que el lenguaje receptivo es mejor que el lenguaje expresivo, aunque en ambos casos se observa retrasos marcados. Al analizar la fonética y fonología utilizada por los pacientes, se nota frecuentes sustituciones, omisiones y distorsiones. El número de consonantes usadas es pequeño y la cantidad de sílabas empleada también es restringida. Sin embargo, recurren a variedad de vocales, aunque se solapan entre sí acústicamente. Se sabe poco acerca de sus habilidades sintácticas, aunque algunas personas pueden expresarse haciendo uso de dos o más palabras para comunicarse (Cornish, Bramble, Munir \& Pigram, 1999; Cornish \& Munir, 1998).

Según Carlin (1988b) el desarrollo de la palabra es posible en como mínimo el $50 \%$ de individuos educados a domicilio y sometidos a una intervención precoz bien organizada. A menudo resulta útil la introducción del lenguaje de gestos. Con el tiempo se puede observar un aumento de la utilización combinada del habla, los signos mediante gestos y medios para-verbales de comunicación. No obstante, se carece de los detalles relacionados con estos desarrollos. De todas formas se cree que los individuos con CDC sólo difícilmente llegan a expresarse únicamente por medio del habla y del lenguaje, aunque cualquier conclusión es prematura dada la escasa perspectiva disponible, porque los refuerzos educativos son muy recientes y el número de casos estudiados muy limitado.

En cuanto al desarrollo académico, la mitad de los niños adquieren destrezas verbales suficientes para comunicarse; si bien, muy pocos lograrán competencias adecuadas en lectura y escritura y si las logran será a un nivel muy básico con frágil permanencia a lo largo del tiempo, necesitando un refuerzo continuado para su mantenimiento. Normalmente, el grado de retraso motor condiciona el desarrollo cognitivo: cuanto mayor es el retraso motor peor es el funcionamiento intelectual y lingüístico (Cornish \& Pigram, 1996). Un fenómeno reiteradamente observado es que los signos fenotípicos se vuelven menos evidentes a media que los chicos se hacen mayores. La certeza de esta información nos llevaría a pensar que el desarrollo motor podría mejorar con la maduración y, por ende, el nivel funcional cognitivo también podría mejorar (Collins \& Eaton-Evans, 2001).

El área de desarrollo social es un aspecto de preocupación de maestros y pediatras. Se sabe que estos niños son capaces de establecer relaciones sociales con sus iguales, que disfrutan con las situaciones de juego, pero no tienen conciencia de los riesgos que implican estas situaciones y ejecutan acciones susceptibles de peligro y daño para su salud física. Cuando emiten juicios sobre las consecuencias de sus actos no son capaces de anticipar los riesgos y los resultados (Cornih, Bramble, Munir \& Pigram, 1999). Según el testimonio de familiares y asociaciones, como aspectos positivos cabe mencionarse que pueden tener una excelente memoria para situaciones o personas de interés especial, así como un sentido del humor muy peculiar. Son buenos imitadores y pueden copiar modelos de otros niños. Otro aspecto destacado en la literatura es su carácter amable con las personas conocidas, 
que no siempre se manifiesta de igual forma con las personas extrañas. Cornish y Pigram (1996) describen otros rasgos clínicos negativos que conviene tener en cuenta a la hora de tratar a estos niños: a) Conducta hiperactiva muy frecuente (50\% de casos), b) Hiperactividad acompañada de agresividad (arañar, morder y golpear objetos y personas); c) Conductas autolesivas; d) Movimientos repetitivos; e) Hipersensibilidad a los sonidos; y f) Apego obsesivo a objetos y personas; g) Conductas desafiantes; h) Miedos injustificados; i) Reacciones anómalas ante extraños; y j) Timidez extrema.

\section{ESPERANZA Y CALIDAD DE VIDA}

En la fase inicial posterior al descubrimiento y descripción del CDC (año 1963), la longevidad de estas personas era un aspecto marcado por cierta imprecisión. En aquel momento, las estimaciones de vida para estos niños eran muy escasas y muchos de ellos morían en los primeros años de vida. Sin embargo, una serie de datos recientes obtenidos por diversos investigadores en estudios longitudinales (Carlin, 1988a, 1988b; Wilkins, Brown \& Wolf, 1980; Wilkins, Brown, Nance \& Wolf, 1982) muestran que el pronóstico actual es claramente mejor por lo que respecta a la salud, al desarrollo psicológico y a la esperanza de vida. Estas indicaciones permiten un mayor optimismo para las personas con CDC y sus familias. El mantenimiento en la familia y la educación precoz parecen constituir los mejores ingredientes de este pronóstico más favorable.

El punto más delicado y que preocupa a las familias es la alta probabilidad de que los niños con CDC sean incapaces de valerse por sí solos y que puedan desenvolverse socialmente. Como ya hemos señalado, sólo la mitad de los niños adquieren las habilidades verbales suficientes para comunicarse.

Existe, sin embargo, la esperanza de que algunos de los síntomas se moderen con el paso del tiempo y sobre todo que los programas de entrenamiento rehabiliten las áreas más deterioradas. En el plano de la salud física, otras preocupaciones están relacionadas con las habituales complicaciones respiratorias y de oído que precisan incluso de ingreso hospitalario (Rizzi, 1997). Muchos padres con hijos afectados destacan que no necesitan dormir mucho, incluidos los recién nacidos.

\section{INTERVENCIÓN PSICOLÓGICA}

El trabajo terapéutico de los CDC debería empezar desde los 3 meses, tomando en cuenta dos vertientes: terapia física para lograr la mayor independencia de movimientos posibles y estimulación temprana para desarrollar sus aptitudes y potencial intelectual. El desarrollo de estas habilidades dependerá del grado y la severidad del síndrome. Uno de los aspectos que el especialista debe considerar es la evaluación de un fisioterapeuta, quien determinará si el niño requiere aparatos para desplazarse e incluso indicará si hay que practicar una intervención quirúrgica para modificar algún defecto. La programación de ejercicios físicos adecuados a las necesidades de estos niños podría ayudarles a mejorar su equilibrio o fuerza en piernas y brazos, de modo que con el tiempo sean capaces de gatear, caminar e incluso correr. Si se da el caso de que el niño exhibe una musculatura tensa o mantiene uno o los dos puños cerrados, se recomienda actividades relajantes para distender esa musculatura. El propósito de este entrenamiento es que los CDC realicen todas aquellas actividades que les otorguen libertad de movimientos y autonomía personal, dentro de las limitaciones que el síndrome impone (Echeverría, 2000).

En cuanto a los problemas cognitivos, a veces se piensa que los CDC ni entienden ni aprenden. Todo depende de la valoración de cada caso, pues existe mucha variabilidad. En algunos casos se debe poner el acento en terapia del lenguaje y en otros en las alteraciones de la atención. De acuerdo a su edad y capacidad se pueden realizar dinámicas y ejercicios que les ayuden a identificar figuras, formas, tamaños, objetos de la casa y alimentos; además de que en la actualidad se trabaja con programas de computación especiales que mejoran su coordinación y memoria. El uso de estos recursos informáticos está resultando muy eficaz para el aprendizaje y a su vez generan niveles de motivación altos. Otra recomendación es el cuidado médico. Muchos de estos niños padecen dificultades cardiacas, problemas digestivos, alteraciones renales y otros problemas neurológicos como crisis convulsivas. 
Los CDC suelen mostrar algunas conductas muy disruptivas (Cerruti Mainardi, 2006) que deben ser intervenidas para dotar a los niños de modelos adecuados de conducta y comunicación (Dykens \& Clarke, 1997). La intervención deberá ser multidisciplinar.

A nivel conductual se recomienda el uso de técnicas de modificación de conducta para reducir y/o eliminar las conductas inadecuadas. Estas técnicas (reforzamiento, extinción, moldeamiento, etc.) deberán adecuarse al nivel y características del niño, por tanto, deberán ser personalizadas. Como parte del tratamiento, los padres deberán ser entrenados para poner en marcha estas estrategias en el ámbito familiar. En el caso de que las manifestaciones conductuales sean potencialmente peligrosas para el propio niño u otras personas, se valorará la posibilidad por un psiquiatra de introducir la medicación pertinente (Clarke \& Boer, 1998). También se deberá intervenir las pautas y hábitos de autonomía como alimentación, higiene, etc.

En el terreno psico-educativo, el área principal de intervención será el abordaje del retraso mental asociado al síndrome (Cornish, Bramble, Munir \& Pigram, 1999; Chang, Lin, Lin, Chen, Kao, Yeung et al, 2007). Las técnicas de estimulación cognitiva y logopédica pueden aportar avances significativos. El juego constituye una estrategia motivadora para la realización de las diferentes actividades. Éstas deben estar encaminadas a conseguir el éxito siguiendo un nivel creciente de dificultad. Se puede trabajar la atención sostenida mediante juegos visuales en el ordenador y utilizar estímulos auditivos si son bien aceptados por el niño. Un objetivo importante es el de establecer un canal comunicativo eficaz con el niño, lo que supondría un paso importante en la prevención de las conductas disruptivas, ya que el niño conseguiría comunicarnos de forma eficaz sus necesidades o deseos, al tiempo que el educador también podría requerirle algunas cosas. Se aconseja (en ausencia de la expresión verbal), la confección de un libro con imágenes o fotografías para la identificación de acciones o personas.

\section{SÍNDROME DOWN: ETIOLOGÍA}

EI SD es una de las causas más comunes de la deficiencia intelectual. La frecuencia de ocurrencia es aproximadamente 1 caso cada 750 nacimientos vivos en la población normal (CDCP, 2006; Roizen \& Patterson, 2003). EI SD es el primero de los síndromes definido por una alteración de tipo numérico, causado por una trisomía en todo o en parte del cromosoma 21 (el más pequeño de los autosomas humanos). La trisomía completa está presente en el $95 \%$ de los casos y sólo un $5 \%$ de los pacientes presentan trisomía parcial por una translocación de un fragmento del cromosoma 21 a los cromosomas 13, 14, 15, 21 o 22 (Devlin \& Morrison, 2004).

En situaciones normales, la unión de un óvulo y un espermatozoide da como resultado un óvulo fertilizado con 46 cromosomas. Sin embargo, algunas veces el proceso ocurre de manera errática; un óvulo o un espermatozoide en desarrollo pueden dividirse de manera incorrecta y producir un óvulo o espermatozoide con un cromosoma 21 de más. Cuando este espermatozoide se une con un óvulo o espermatozoide normal, el embrión resultante tiene 47 cromosomas en lugar de 46 (Lamb \& Hassold, 2004). Las causas de los fallos son muy variadas, una de las más significativas es la exposición a determinados productos químicos o virus que afectan a la calidad del esperma y los óvulos, otra es cierto estilo de vida excesivamente estresante o el uso de determinadas máquinas o prendas de vestir.

Ocasionalmente, antes de la fertilización, una parte del cromosoma 21 se desprende durante la división celular y se adhiere a otro cromosoma dentro del óvulo o del esperma. El embrión resultante puede tener lo que se conoce como SD con translocación. Las personas afectadas tienen dos copias normales del cromosoma 21 más material del cromosoma 21 adicional adherido a otro cromosoma. Este tipo de accidente en la división celular es responsable de aproximadamente del $35 \%$ al $45 \%$ de los casos de SD. En algunos casos, el padre o la madre tiene una redistribución del cromosoma 21, llamada translocación equilibrada, que no afecta su salud. Aproximadamente del $1 \%$ al $2 \%$ de las personas tiene una forma de SD llamada SD en mosaico (Patterson \& Lott, 2008). En este caso, el accidente en la división celular tiene lugar después de la fertilización. Las personas afectadas tienen algunas células con un cromosoma 21 adicional y otras con la cantidad normal. 
El origen de la no-disyunción en el SD es maternal en el 95\% de los casos y la incidencia de nacimientos con SD se incrementa de manera sorprendente a medida que aumenta la edad de la madre (Lamb \& Hassold, 2004). Cuando la madre tiene menos de 29 años, la probabilidad es $1 / 3000$, entre 30 y 34 es $1 / 2000$, entre 35 y 39 es $1 / 280$, entre 40 y 44 es $1 / 70$ y entre 45 y 49 es $1 / 40$. Aunque el riesgo es mayor a medida que aumenta la edad de la madre, aproximadamente el $80 \%$ de los bebés con SD nacen de mujeres de menos de 35 años, ya que las mujeres más jóvenes tienen más bebés que las mujeres de mayor edad (CDCP, 2009).

En términos generales, las probabilidades de concebir otro bebé con SD en cada embarazo subsiguiente son las siguientes: con menos de 29 años de edad de la madre la probabilidad es $1 / 1000$, entre 30 y 34 es $1 / 800$, entre 35 y 39 es $1 / 100$, entre 40 y 44 es $1 / 25$ y entre 45 y 49 es $1 / 15$ (Morris, Mutton \& Albergan, 2005). Sin embargo, si el primer niño presenta SD con translocación, las probabilidades de tener otro niño con SD pueden incrementarse enormemente. La reciente investigación ha demostrado que la no-disyunción puede ocurrir no sólo con el cromosoma 21 si no que también puede suceder con otros cromosomas y que algunas mujeres son más proclives que otras a la ocurrencia de este fenómeno anómalo (Warburton et.al, 2004).

Por lo general, después del nacimiento, cuando se sospecha que un bebé tiene SD, el médico le extrae una muestra de sangre para realizar un análisis cromosómico (Ilamado cariotipo). Este análisis sirve para determinar si el bebé tiene SD y para identificar la forma genética correspondiente. Esta información es importante para determinar el riesgo que corren los padres en futuros embarazos. El médico podrá enviar a los padres a un asesor en genética para que les explique detalladamente los resultados de este análisis cromosómico y les indique cuáles son los riesgos de que se repita este fenómeno en otro embarazo.

\section{DIAGNÓSTICO}

El diagnóstico del SD antes de que nazca un bebé es una preocupación de muchas parejas. El Colegio de Obstetras y Ginecólogos de los Estados Unidos (American College of Obstetri- cians and Gynecologists, ACOG) recomienda ofrecer a todas las mujeres embarazadas, independientemente de su edad, una prueba de detección precoz de SD. La prueba puede consistir en un análisis de sangre de la madre durante el primer trimestre (entre las semanas 11 a 13 del embarazo) junto con un examen especial por ultrasonidos para medir el grosor de la nuca del bebé (translucencia nucal), o bien en un análisis de la sangre materna durante el segundo trimestre (entre las semanas 15 a 20) sin el ultrasonido (ACOG, 2007). Las pruebas de detección precoz ayudan a identificar los embarazos con un riesgo de SD superior al promedio pero no pueden diagnosticar éste $u$ otros defectos congénitos.

Las mujeres que obtienen un resultado anormal en la prueba pueden realizar una prueba de diagnóstico, como una amniocentesis (Steele \& Bregg, 1966) o una muestra del villus coriónico (CVS) (Simoni et.al. 1983). Estas pruebas permiten diagnosticar o, más frecuentemente, descartar la existencia de SD con gran exactitud $(0,5 \%-1 \%$ de riesgo según Brambati \& Tului, 2005). El procedimiento CVS ofrece una ventaja considerable con respecto a la amniocentesis es que se puede aplicar al término del primer trimestre, cuando el riesgo de mortalidad de la madre es de 1 en 100.000 casos, en oposición a lo que ocurre al término del segundo trimestre donde el riesgo se eleva a 9 casos en 100.000 que es cuando se utiliza la amniocentesis (Pinette et.al, 2004).

Las pruebas de detección precoz o de diagnóstico sirven para tranquilizar a la mayoría de los padres de que su bebé no tiene SD. No obstante, si la prueba de diagnóstico prenatal muestra que el bebé tiene $S D$, los padres tienen la oportunidad de considerar el diagnóstico y sus opciones, así como de prepararse médicamente, emocionalmente y financieramente para el nacimiento de un niño con necesidades especiales y programar el parto en un centro médico debidamente equipado.

La mayoría de los problemas de salud asociados con el SD puede tratarse y la expectativa de vida es actualmente de unos 60 años (NDSS, 2009). Las personas con SD tienen más probabilidades que las personas no afectadas de tener una o más de las siguientes enfermedades: Defectos cardíacos $(50 \%)$, de- 
fectos intestinales (12\%), problemas de visión $(60 \%)$, pérdida auditiva $(75 \%)$, infecciones y resfriados $(20 \%)$.

\section{CARACTERÍSTICAS FÍSICAS Y MÉDICAS}

Los niños con SD tienen una apariencia característica ampliamente reconocida, con una cabeza que puede ser más pequeña de lo normal (microcefalia) y deformada. Los rasgos faciales prominentes son nariz achatada, lengua protuberante y ojos inclinados hacia arriba. La esquina interna del ojo puede tener un pliegue redondeado de piel (pliegue del epicanto) en lugar de terminar en punta. Las manos son cortas y anchas con dedos cortos, que suelen tener un único pliegue palmar (pliegue simiano). El retraso en el crecimiento y desarrollo es característico y la mayoría de los niños afectados nunca alcanzan una altura adulta promedio.

Los defectos cardíacos congénitos en estos niños son frecuentes y la muerte prematura (en el pasado) a menudo se presenta como resultado de estas anomalías cardíacas. Las anomalías gastrointestinales, como la atresia esofágica (obstrucción del esófago) y la atresia duodenal (obstrucción del duodeno) también son relativamente comunes. La obstrucción del tracto gastrointestinal puede requerir una cirugía poco después del nacimiento. Además pueden presentar: disminución del tono muscular al nacer, suturas craneales separadas, cráneo asimétrico o deforme, cabeza redonda con un área plana en la parte de atrás (occipital), boca pequeña, manos cortas y anchas, lesión en el iris, perfil facial plano, orejas pequeñas, músculos abdominales separados, articulaciones hiperflexibles, marcha inestable, piel de más en la parte posterior del cuello al nacer y un hueso anormal en la mitad del quinto dedo, problemas de visión, pérdida auditiva, infecciones de oído frecuentes y en general mayor susceptibilidad a infecciones, obstrucción gastrointestinal, la tercera parte de los pacientes experimenta bloqueo de las vías respiratorias durante el sueño, aumento en la incidencia de demencia en pacientes mayores, inestabilidad de los huesos de la espalda en la parte superior del cuello que puede provocar lesiones compresivas de la médula espinal (Shapiro, 1983).

\section{DESARROLLO PSICOMOTOR}

La literatura existente sobre el desarrollo psicomotor de los SD ha puesto de manifiesto una serie de peculiaridades inherentes a la trisomía-21 que pueden explicar la demora que experimentan estos niños. La característica más relevante cuando se habla de desarrollo psicomotor en niños con SD es la hipotonía. Aunque los efectos y el grado de hipotonía son variables, se ha visto relación entre ésta y los problemas para el reconocimiento táctil, cutáneo y kinestésico, como consecuencia de las alteraciones que dicha hipotonía produce a nivel del sistema nervioso central, fundamentalmente en las áreas sensoriales. En este sentido, se observan problemas perceptivos visuales, auditivos y kinestésicos que afectan a todas las conductas que implican movimiento, tales como la coordinación dinámica y óculomanual, así como la percepción espacio-temporal (Harris, 1984).

La laxitud de las articulaciones es otra de las características más comunes debido a la hipotonía muscular, que repercute tanto en el movimiento como en la postura. La inestabilidad atlanto-axial y los problemas de la rodilla son factores que contribuyen a las dificultades para mantener el equilibrio y la inseguridad e inmadurez de los patrones locomotrices. Otra de las peculiaridades que podría explicar las dificultades y diferencias motrices de estos niños ha sido la morfología del pie, cuyas alteraciones, si no son tratadas, pueden ocasionar problemas en la coordinación y el equilibrio.

Las diversas investigaciones sobre el desarrollo psicomotor de los niños con SD (Block, 1991), destacan como resultados en sus estudios que: a) Algunos niños con SD presentan una demora considerable en el desarrollo motor. Por ejemplo, la adquisición de la marcha no se suele producir antes de los dos años. b) En el primer año de vida, los niños con SD manifiestan patrones de movimiento y estrategias diferentes cuando se comparan con niños cuyo desarrollo es normal. c) Asimismo, estos niños presentan mayor lentitud en la aparición y disolución de reflejos y modelos automáticos de movimiento. 


\section{EVOLUCIÓN COGNITIVA}

Una característica definitoria del SD es que siempre se acompaña de deficiencia mental en diferentes grados (el Cl varía desde 20 hasta 60 , Benedet, 1991; NDSS, 2009; AAPCG, 2007). El $\mathrm{SD}$, como grupo, se mueve en la actualidad en el rango de la deficiencia ligera o media, con algunas excepciones por arriba (capacidad intelectual "límite") y por abajo (deficiencia severa y profunda), estas últimas debidas en la mayor parte de los casos a una estimulación ambiental limitada más que a carencias constitucionales. Entre los factores específicos de los grados de inteligencia mencionados, puede un sujeto con SD destacar en uno de ellos respecto a sus compañeros (por ejemplo, en el factor verbal) enmascarando su baja capacidad en otros. $\mathrm{O}$ a la inversa, una persona con muchas dificultades de expresión lingüística puede tener mayor capacidad intelectual que otra que se exprese con más claridad (AAMR, 1997).

La afectación puede ser muy distinta en cada uno de los campos, sin que necesariamente se dé una relación directa entre unos y otros. En los tests estandarizados alcanzan mejores puntuaciones en las pruebas manipulativas que en las verbales, lo que como conjunto penaliza a la población con SD, ya que el contenido lingüístico de la mayor parte de las baterías de tests es muy alto. También se observa que, al igual que la mayoría de las personas con retraso mental, las puntuaciones globales en las pruebas de inteligencia descienden de manera drástica cuando se acercan a la adolescencia. Esto se debe a que en esta edad la población general adquiere el denominado pensamiento formal abstracto, con el cual las personas con SD tienen especiales dificultades. De hecho, en los primeros años de vida, al aplicar pruebas de desarrollo a niños estimulados, las puntuaciones obtenidas no varían en exceso respecto a la población sin deficiencia. Sin embargo, con el paso del tiempo el desnivel respecto a la población general se hace cada vez más marcado (Brown, Greer, Aylward \& Hunt,1990; Robert, Chapman \& Warren, 2008).

La pérdida de memoria es uno de los síntomas que últimamente acumula más investigación en estos pacientes. Las personas con SD son más propensas que las personas no afectadas a desarrollar la enfermedad de Al- zheimer (caracterizada por la pérdida gradual de la memoria, cambios en la personalidad y otros problemas). Las recientes investigaciones confirman que la gran mayoría de los SD a la edad de 40 años de edad muestran signos de neuropatología tipo Alzheimer (Mann \& Esiri, 1989). Los cambios cerebrales observados ponen de manifiesto la presencia de fragmentos de proteína beta-amiloide, rodeados de neuronas en proceso de degeneración. En estos enfermos, las placas malignas se concentran en ciertas partes del cerebro, como el lóbulo temporal medio (hipocampo, amígdala, córtex) y en el lóbulo parietal posterior (Leverenz \& Raskind, 1998). Los adultos con SD suelen desarrollar la enfermedad de Alzheimer antes que las personas no afectadas (Tyrrell et.al., 2001). Los estudios sugieren que aproximadamente el 25 por ciento de los adultos con SD de más de 35 años tienen síntomas de enfermedad de Alzheimer (Wisniewski, Wisniewski \& Wen, 1985).

\section{EVOLUCIÓN DEL LENGUAJE}

Algunos investigadores de los noventa se han interesado en explorar el desarrollo del lenguaje de los SD y compararlo con otros sujetos que padecen enfermedades raras y presentan un retardo mental similar. Según estos investigadores (Rondal, 1988a, 1994a y Fallase, Comblain y Rondal 1994) todos los individuos con un retardo mental moderado presentan deficiencias en el área de la morfosintaxis. Es posible que los SD estén especialmente desfavorecidos desde este punto de vista pero-en comparación con otros RM con los mismos niveles de incapacidad y la misma EM - es únicamente una variación cuantitativa y no un desarrollo o un funcionamiento morfosintáctico cualitativamente diferente.

Un problema complejo en estos estudios tiene que ver con los grupos de comparación ( $\sin$ SD). Se trata de grupos de participantes con RM, cuya etiología es desconocida o mixta. Una heterogeneidad de este tipo no permite, en un sentido estricto, establecer que el SD presente más desventajas para el lenguaje que las otras entidades patológicas que conducen a un RM. De hecho, se ha observado que algunos individuos en las diferentes investigaciones, muestran un rendimiento lingüístico similar al de las personas con SD. Con esto se deduce que la 
capacidad lingüística en el SD no es prototípica del retraso mental moderado o severo. Se puede deducir que las diferencias de lenguaje entre los síndromes de RM provienen de diferencias a nivel cerebral que probablemente tengan su origen en la diversidad genética característica de estos síndromes.

La inferioridad lingüística de los SD (suponiendo que sea real) se relaciona sobre todo con componentes de la fonético-fonología (articulación, discriminación auditiva, inteligibilidad del habla) y de la morfosintaxis (longitud media del enunciado, marcadores morfológicos, organización sintáctica). Sin duda, es inadecuado definir esta inferioridad como «lingüística» cuando las deficiencias se relacionan principalmente con los componentes "computacionales» del lenguaje (Chomsky, 1981) y no, o mucho menos, con los componentes conceptuales (léxico, semántica estructural y pragmática del lenguaje) (Fowler, 1988; Rondal, 1988a, 1988b). Incluso si los SD presentaran una cierta inferioridad en el habla y la morfosintaxis con respecto a muestras mixtas de individuos RM (equiparables en nivel mental), este tipo de comparación en último término sería poco informativa. Es mucho más interesante realizar comparaciones bien controladas de grupos e individuos RM con etiologías perfectamente definidas.

\section{DEFICIENCIAS FONOLÓGICAS Y ATENCIONALES}

El desarrollo auditivo es uno de los problemas más frecuentes en niños con SD debido a infecciones que causan pérdida de la audición en los primeros años de vida (Robert \& Medly, 1995). Lynch Oller, Steffens y Levine (1995) han descubierto que 6 de 13 niños pequeños con SD examinados habían perdido entre 25$40 \mathrm{~dB}$ debido a otitis media. Las deficiencias auditivas pueden repercutir negativamente en la adquisición del lenguaje. Existe un amplio consenso entre los investigadores que señalan que el desarrollo fonológico es fundamental para la adquisición de habla y la comunicación (Roberts, Chapman \& Warren, 2008).

Los niños con SD muestran un desarrollo motor que generalmente afecta el desarrollo del habla. Entre los impedimentos que podemos mencionar se encuentran: el bajo tono muscular de la lengua, los labios y mejillas ocasionando producciones de sonidos del habla poco firmes y precisos (Kumin, 1996). Se sabe que los SD tienen un paladar alto, estrecho y arqueado acompañado de amígdalas y adenoides alargadas, boca y mandíbula pequeña en comparación con el tamaño de la lengua (Strome \& Strome, 1992). Todas estas características físicas combinadas contribuyen a la dificultad de los SD para producir sonidos y combinaciones de sonidos, aspecto éste necesario para entender el habla.

El control de la atención es un problema para los SD, aunque ellos sean muy sociables (Wishart \& Johnston, 1990). Una de las mayores dificultades tiene que ver con el contacto ocular y la reciprocidad del contacto (Berger \& Cunningham, 1983). Cuando los investigadores compararon el desarrollo del contacto visual con sus cuidadores en los SD y en los niños de un grupo control, encontraron que el grupo control aceleraba su reciprocidad de contacto visual en 10 semanas aproximadamente. Además comprobaron que una vez que los SD establecían el contacto visual tendían a mantenerlo de forma más extendida en el tiempo, lo que llevaría a concluir que estos niños muestran un bajo nivel de exploración hacia otros aspectos de la interacción (Berger, 1990; Kasari, Mundy, Yirmiya, \& Sigman, 1990). Los autores hacen notar que esta deficiencia en la exploración y evaluación del entorno podría enmascarar la capacidad de los SD para adquirir las habilidades del lenguaje.

\section{LENGUAJE DE SIGNOS Y CONDUCTA IMITATIVA}

El uso de gestos, como forma de comunicación, comienza a la edad de 12 meses. Caselli y Casadio (1998) examinaron el lenguaje signado y gestual de 40 niños italianos con SD. Los investigadores compararon sujetos $S D$ con otro grupo normativo. Los resultados confirman que los SD usan más gestos que el grupo control. Esta diferencia sólo apareció cuando ambos grupos habían adquirido un nivel léxico mínimo de 100 palabras. Los SD usan más gestos sobre todo cuando quieren algo (por ejemplo: "jugar con la muñeca") o en conductas comunicativas cuyo símbolo es muy gestual (por ejemplo: "ir", "caliente", "estar quieto"). 
Caselli y Casadio sugieren que los niños SD de su estudio parecen estar especializados en comunicación no verbal, posiblemente debido a que han estado expuestos más a este lenguaje que los niños del grupo control.

La capacidad de imitación es una de los aspectos positivos más destacables de los niños SD (Rast \& Meltzoff, 1995). Wright, Lewis y CoIlis (2006) examinaron la imitación en un grupo de niños de 11 y 43 meses y la compararon con otro grupo control de la misma edad. Los autores descubrieron que los niños SD usaban la imitación con mayor frecuencia en tareas de búsqueda de objetos y juegos que los niños del grupo control.

\section{CONCLUSIONES}

Los SD y CDC muestran algunas semejanzas y diferencias específicas. Una diferencia clara es la prevalencia del síndrome. En el SD la prevalencia es un 1:750 nacimientos vivos, una prevalencia que según los informes epidemiológicos es relativamente alta y que puede incrementarse en función de la edad de la madre, como señalan todos los estudios desde hace algún tiempo. Diferente es la situación con el CDC donde los estudios indican que la frecuencia de aparición de la enfermedad es 1:50.000 recién nacidos vivos. Se trata pues de una prevalencia muy baja. En efecto, es una de las más bajas en el cuadro de las enfermedades consideradas raras.

Los estudios genéticos han permitido descubrir que ambos síndromes tienen una etiología genética, relacionada con una alteración cromosómica. EI SD proviene de una alteración cromosómica de tipo numérico en el par 21 con consecuencias múltiples, siendo la principal la afectación negativa de las áreas cognitivas, con particular incidencia en la función intelectual. El CDC también es una alteración cromosómica de naturaleza estructural que se localiza en el par 5 y se produce por delección, translocación o pérdida parcial en el brazo corto del cromosoma. El cariotipo en ambos casos es una prueba que permite reconocer el mapa cromosómico y su configuración anómala de forma precoz para de esta manera prepararse y actuar lo antes posible. En los CDC, la magnitud de los daños estructurales determinará las zonas concretas afectadas y circunscribirá el nivel de funcionamiento biológico y cognitivo.

Entre los rasgos comunes compartidos encontramos el retardo mental. En ambos casos el síndrome se expresa con deficiencia mental, que oscila entre moderada y grave, con predominio moderada. Sin embargo, en el CDC la manifestación de retardo puede llegar a ser severa, incapacitando a los afectados en la autonomía personal. La severidad del retardo en el CDC viene marcada por la cantidad de delección cromosómica producida en cada uno de los afectados. La variabilidad hace que la severidad del retardo cambie entre unos y otros, haciendo difícil la predicción del rendimiento intelectual. El funcionamiento intelectual de los Down es más homogéneo entre los afectados: es infrecuente encontrar niños que superen un $\mathrm{Cl}$ de 70 y que bajen de 40 . El valor promedio está en 50 de $\mathrm{Cl}$ en las pruebas estándar de inteligencia. Hoy en día según AAMR se reconoce abiertamente que el $\mathrm{Cl}$ es una condición necesaria pero no suficiente para definir una persona con retardo mental.

Así como la inteligencia funcional de los SD y CDC se ha evaluado de forma precisa y consistente, el perfil lingüístico de ambos es impreciso, principalmente con los CDC. Las posibilidades de desarrollo del lenguaje de los CDC a penas si se conocen y la información conocida procede de la descripción de un puñado de casos que ofrece escaso consenso en las valoraciones y, por ende, es difícil llegar a conclusiones válidas. La literatura señala que los sujetos CDC muestran carencias a nivel lingüístico que generalmente suelen ser graves: retraso en el habla y desarrollo de la palabra y el discurso limitados; aunque se reconoce que las investigaciones científicas acerca del tema todavía está en fases no muy avanzadas.

El perfil lingüístico de los SD es mejor conocido y sus características vienen definidas por observaciones sistemáticas con muestras amplias de sujetos y trabajos de campo controlados. Los principales problemas lingüísticos de los sujetos SD son los siguientes: a) Dificultades articulatorias con algunos fonemas; b) Maduración de la discriminación fonémica lenta y a veces incompleta; c) Léxico reducido tanto en el número de lexemas como en las características semánticas dentro de los lexemas; 
d) Escasa organización del léxico mental, tanto a nivel semántico como gramatical; e) Reducción en la complejidad formal y longitud de las elocuciones; f) Problemas con la producción y comprensión de proposiciones subordinadas y oraciones complejas; g) Problemas con la morfología inflexional; $h$ ) Lentitud en el desarrollo de habilidades pragmáticas e; i) Desarrollo de macroestructuras discursivas insuficiente.

El patrón de rasgos físicos es muy diferente en ambos síndromes. El de los SD es perfectamente reconocible y consistente a lo largo del tiempo, cosa que no siempre ocurre con los CDC que pueden cambiar a lo largo del ciclo vital, dando lugar a imprecisiones. Cuando estos niños nacen tienen un tono muy agudo al llorar que resulta poco común al escucharlo, Sin embargo, hay que aclarar que este síntoma desaparece pasados 3 ó 4 meses dando lugar a un llanto normalizado. Algo similar ocurre con otro rasgo físico "la cara de luna" que generalmente se asocia a niños CDC como rasgo diferenciador. Como en el caso anterior, dicho rasgo se suaviza con el tiempo y aproximadamente en el segundo trimestre se regulariza. La falta de una pequeña porción de cromosoma en el par 5 genera bajo peso al nacer, baja estatura, debilidad muscular, problemas al caminar, disfunciones cardiacas y respiratorias entre otras características físicas. Aunque la literatura así lo señala de forma genérica, cada caso ofrece un conjunto de rasgos que son diferentes. Por ejemplo, la estatura no siempre es baja, los estudios clínicos confirman algunos casos que pueden llegar a medir 1.80 .

El promedio de vida de las personas con CDC está disminuida, aunque la mayoría alcanza la edad adulta (alrededor de 50 años), a menos que nazca con otras malformaciones severos en alguno de los órganos vitales (por ejemplo, cardiopatías). La pérdida de memoria es uno de los síntomas cognitivos más investigados en los SD. Hoy se sabe que estas personas son más propensas que las personas no afectadas a desarrollar la enfermedad de Alzheimer. Las recientes investigaciones confirman que la gran mayoría de los SD a la edad de 40 años de edad presentarán signos de demencia senil y demencia tipo Alzheimer. La biología de la memoria en sujetos CDC no se conoce y no parece existir investigaciones al respecto. Probablemente un factor que lo explica es que la esperanza de vida de los CDC es más corta y los efectos perniciosos de la enfermedad no se manifiestan de manera tan nítida como ocurre con los SD. Aunque no existan datos que lo puedan confirmar, los investigadores asumen que las deficiencias en la memoria correrán de forma paralela al deterioro mental, lingüístico $y$ atencional.

En resumen, conviene remarcar que los SD y CDC comparten algunos rasgos. Primero, ambos se originan en una anomalía cromosómica pero diferente, y cada uno cursa con alteraciones específicas. Segundo, ambos muestran retraso intelectual y psicomotor, el $\mathrm{Cl}$ oscila entre moderado y severo. En los CDC se da con mayor frecuencia el $\mathrm{Cl}$ bajo y en los SD el moderado. Tercero, ambos comparten problemas médicos como cardiopatías y alteraciones musculares y esqueléticas y problemas auditivos y visuales. Cuarto, el bajo tono muscular es muy marcado en los CDC y frecuente en los SD. Quinto, los CDC presentan un retraso comunicativo importante, en los SD no es tan significativo. Comparten sin embargo un aspecto deficitario del lenguaje: la fonología. Debido a la influencia de factores anatomofisiológicos, el desarrollo fonológico es lento y difícil. Entre los fonemas que presentan mayores problemas encontramos los fricativos ( $f, z, s, y)$, los laterales $(I, I I)$ y los vibrantes ( $r$ ). En general, la sílaba es menos inteligible que la del resto de los niños más pequeños que no presentan deficiencia mental. Además también son frecuentes los problemas de ritmo (habla muy rápida o tartamudez) (Rondal, 1988a). Los rasgos físicos de los SD son muy estables y definidos mientas que en los CDC son variables y cambian con el tiempo. En general, el conocimiento científico de los SD es amplio y bastante preciso en cuanto al funcionamiento cognitivo, lingüístico y su afectación neurológica, mientras que en los CDC la información acerca de esas áreas es escasa, con muchas zonas grises y oscuras.

\section{REFERENCIAS}

AAMR(1997). Retrasomental. Definición, clasificaciónysistemas de apoyo. Madrid, Alianza Editorial (Psicología). American Academy of Pediatrics Committee on Genetics (AAPCG actualizado, Sept. 2007). Health Supervision for Children with Down Syndrome. Journal of Pediatrics, 107, 2, 442-449. 
Álvarez, R., Chima, M., Madrid, V., Gálvez, E., Rivera, M. \& Cervantes A. (2003). Síndrome de cri du chat: presentación de dos casos. Revista Médica del Hospital General de México, 66:212-7.

American College of Obstetricians and Gynecologists (ACOG, actualizado, Enero, 2007), Screening for Fetal Chromosomal Abnormalities. ACOG Practice Bulletin, 77.

Benedet, M. J. (1991). Procesos cognitivos en la deficiencia mental. Madrid, Editorial Pirámide (Psicología).

Berger, J. (1990). Interactions between parents and their infants with Down syndrome. En D. Cicchetti \& M. Beeghly (Eds), Children with Down syndrome: A developmental perspective (pp. 101-146). New York: Cambridge University Press.

Block, M. E. (1991). Motor development in children with Down syndrome: A review of the literature. Adaptative Physical Activity Quarterly, 8, 179-209.

Brambati, B. \& Tului, L. (2005). Chorionic villus sampling and amniocentesis. Current Opinions in Obstetrisc and Gynecology, 17, 197-201.

Breg, W. R., Steele, M. W., Miller, O. J., Warburtonb, D., Capoa, A. \& Allerdice, P. W. (1970). The cri du chat syndrome in adolescents and adults: clinical finding in 13 older patients with partial deletion of the short arm of chromosome $N^{\circ} 5$ (5p-). Journal of Pediatrics, 77:782-791.

Brown, F. R. III., Greer, M. K., Aylward, E. H. \& Hunt, H. H. (1990. Intellectual and adaptive functioning in individuals with Down syndrome in relation to age and environmental placement. Journal of Pediatrics, 85, 450-452.

Cammarata, F., Araque, D., Da Silva, G. Y. \& Hernández, J. (2009). Síndrome de cri-du-chat. Reporte de dos casos y revisióndelaliteratura. CanariasdePediatría,33(1):7-13.

Carlin, M. (1988a). Longitudinal data shows improved prognosis in Cri-du-chat syndrome. American Journal of Human Genetics, 41, A50.

Carlin, M. (1988b), The improved prognosis in Cri-du-cha (5p-) syndrome. En W. Fraser, W. (Ed), Key issues in mental retardation research (pp. 64-73). London: Routledge.

Caselli, M. C. \& Casadio, P. (1995). II primo vocabulario del bambino. Milano, Franco Angeli.

Center for Disease Control and Prevention (CDCP, 2006), Improved national prevalence estimates for 18 selected major birth defects-United Status, 1999-2001. Morbility and Mortality Weekly Report, 54, 1301-1305.

Centers for Disease Control and Prevention (CDCP, 2009). Down Syndrome. Creado 11 de marzo, www.cdc.gov/ ncbdd/birthdefects/DownSyndrome.htm.

Cerruti Mainardi P. (2006). Cri du Chat syndrome. Orphanet Journal of Rare Diseases, 1:33.

Cerruti Mainardi, P. Cri-du-chat syndrome. Orphanet encyclopedia. Recuperado en octubre de 2003, en http://www.orpha.net/data/patho/GB/uk-criduchat.pdf

Cerruti Mainardi, P., Perfumo, C., Calì, A., Coucourde G., Pastore, G., Cavani, S., et al. (2001). Clinical and molecular characterization of 80 patients with $5 p$ deletion: genotype-phenotype correlation. Journal of Medical Genetics, 38:151-8.
Cerruti Mainardi, P., Guala, A., Pastore, G., Pozzo, G., Dagna Bricarelli, F., \& Pierluigi, M. (2000). Psychomotor development in cri du chat syndrome. Clinical Genetics 57:459-461.

Ceruti Mainardi, P.,Medolago, M. \& Pedrinazzi(2002). La sindrome del cri-du-chat. Grafide Borri, S. Casciano V. P. (firenza).

Clarke, D. J. \& Boer, H. (1998). Problem behaviours associated with deletion Prader-Willi, Smith-Magenis, and Cri du Chat Syndromes. American Journal of Mental Retardation, 103:264-271.

Chang, C. Y., Lin, S. P., Lin, H. Y., Chen, Y. J, Kao, H. A., Yeung, C.Y., et al. (2007). Cri du chat syndrome. Acta Paediatrica Taiwanica, 48:328-31.

Chomsky, N. (1981). Lectures on government and binding. Dordrecht:Foris.

Choong, Y. F., Watts, P., Little, E. \& Beck, L. (2003). Goldenhar and cri-du-chat syndromes: a contiguous gene deletion syndrome? J AAPOS, 7:226-227.

Collins, M. S.\& Eaton-Evans, J. (2001). Growth study of cri-du-chat syndrome. Archives of Diseases in Child, 85, 337-338.

Cornish, K. M. \& Pigram, J. (1996). Developmental and behavioural characteristics of cri-du-chat syndrome. Archives of Diseases in Child, 75, 448-440.

Cornish, K. M. \& Munir, F. (1998). Receptive and expressive speech skills in children with cri-du-chat syndrome. Journal Communication Disorders, 31:73-80.

Cornish, K. M., Bramble, D., Munir, F. \& Pigram, J. (1999). Cognitive functioning in children with typical cri du chat (5p-) syndrome. Developmental Medicine and Child Neurology 41(4):263-6.

Devlin, L. \& Morrison, P. J. (2004). Mosaic Down's syndrome prevalence in a complete population study. Archives of Disease of Childhood, 14, 1177-3749.

Duarte, A.C., Cunha, E, Roth, J. M, Ferreira, F. L, Garcias, G. L.\& Martino-Roth, M. G. (2004). Cytogenetics of genetic counseling patients in Pelotas, Rio Grande do Sul, Brazil. Genetics and Molecular Research, 3:303308.

Dykens, E. M. \& Clarke, D. J. (1997). Correlates of maladaptive behaviour in individuals with $5 p$ - (cri du chat) syndrome. Develomental Medicine and Child Neurology, 39:752-756.

Echeverría, A. (2000). Síndrome de "maullido de gato", guía para padres y educadores. Santander: Consejería de Sanidad, Gobierno de Catabria.

Fang, J. S., Lee, K. F., Huang, C. T., Syu, C. L., Yang, K. J., Wang, L. H., et al. (2008). Cytogenetic and molecular characterization of three-generation family with chromosome $5 p$ terminal deletion. Clinical Genetics, 73:585-590.

Fayasse, M.; Comblain, A., \& Rondal, J. A. (1994). Aspectos morfosintácticos avanzados del lenguaje de niños y adolescentes con retraso mental leve y moderado. Revista de Logopedia, Foniatría y Audiología, 14, 207 218.

Fowler, A. (1988). Determinants of rate of language growth in children with Down syndrome. En L Nadel (Ed.), The 
psychobiology of Down syndrome (pp. 217-245). Cambridge, MA: MIT Press.

Harris, S. R. (1984). Down syndrome. En S. K. Campbell (Ed.) Paedriatric neurologic physical therapy (pp. 169204). Edinburgh. Churchill Livingston.

Kasari, C., Mundy, P., Yirmiya, N \& Sigman, M. (1990). Affect and attention in children with Down syndrome. American Journal of Mental Retadation, 95, 55-67.

Kumin, L. (1996). Speech and language skills in children with Down syndrome. Mental Retardation and Developmental Disabilities, 2, 109-115.

Lamb, N. E. \& Hassold, T. J. (2004). Nondisjuntion: A review from ringside. New England Journal of Medicine, 351, 1931-1934.

Lejeune, J., Lafourcade, J., Berger, R., Vialatte, J., Boeswillwald, M., Seringe, P., et.al. (1963). Trois cas de deletion partielle du bras court d' un chromosome 5. Comptes Rendus Hebdomadaires des Séances de I'Académie Sciences, 257: 3098-102.

Leverenz, J. B. \& Raskind, M. A. (1998). Early amyloid deposition in the medial temporal lobe of young Down syndrome patients: A regional quantitative analysis. Experimental Neurology, 150, 296-304.

Lynch, M. P., Oller, D. K., Steffens, M. L. \& Levine, S. L. (1995), Onset of speech-like vocalization in infants with Down syndrome. American Journal on Mental Retardation, 100, 68-86.

Marinescu, R.C., Cerruti Mainardi, P., Collins, M. R., Kouahou, M., Coucourde, G., Pastore, G., Eaton-Evans, J. \& Overhauser, J. (2000). Growth charts for cri-du-chat syndrome: an international collaborative study. American Journal of Medical Genetcs, 94:153-162.

Mann, D. M. \& Esiri, M. M. (1989). The pattern acquisition of plaques and tangles in the brains of patients under 50 years of age with Dow's syndrome. Journal of Neurological Science, 89, 169-179.

Morris, J. K., Mutton, D. E. \& Alberman, E. (2005). Recurrences of free trisomy 21: Analysis of data from the $\mathrm{Na}$ tional Down Syndrome Cytogenetic Register. Prenatal Diagnosis , 25, 1120-1128.

National Down Syndrome Society (NDSS). Information Topics. Recuperado en abril de 2009, de www.ndss. org.

National Institute of Child Health and Human Development (NICHD) Facts About Down Syndrome. Recuperado en agosto de 2008, de. http://www.nichd.nih.gov/publications/pubs/downsyndrome.cfm.

Niebuhr, E. (1978). The cri du chat syndrome. Epidemiology, cytogenetics and clinical features. Human Genetics, 44:227-275.

Niebuhr, E. (1979). Antropometry in the Cri du Chat syndrome. Clinical Genetics, 16:82-95.

Patterson, D \& Lott, I. (2008). Etiology, diagnosis and development in Down Síndrome. En J. E Roberts, R. S. Chapman \& S. F. Warren (Eds), Speech, Language and Intervention in Down Syndrome and Fragile $X$ Syndrome. Baltimore, Paul H. Brookes Publishing Co.

Pinette, M. G., Wax, J., Blackstone, J., Cartin, A. \& McCran n, D. (2004). Timing of early amniocentesis and a function of membrane fusion. Journal of clinical Ultrasound, 43, 8-11.

Rast, M. \& Meltzoff, A. N. (1995). Memory and representation in young children with Down syndrome: Exploring deferred imitation and object permanence. Development and Psychopathology, 7, 393-407.

Rizzi, M. (1997). Valutazione immunologica in pazienti affetti dalla sindrome del cri du chat $5 \mathrm{p}$-. In Tesi di Laurea. Facoltà di Medicina e Chirurgia, Università degli Studi di Milano, Anno Accademico.

Robert, J. E., Chapman R. S. \& Warren, S. F. (2008). Speech, language and development intervention in Down syndrome and Fragile $X$ syndrome. Baltimore, Paul H. Brookes Publishing.

Robert, J. E. \& Medley, L (1995). Otitis media and speechlanguage sequelae in young children: Current issues in management. American Journal of Speech-Language Pathology, 4, 15-24.

Rogers, P. T. (1994). Coleman M. Atención médica en el Síndrome de Down: Un planteamiento de medicina preventiva. Barcelona, Fundació Catalana Síndrome de Down.

Rondal, J.A. (1988a). Down's syndrome. En D. Bishop y K. Mogford(Eds), Languagedevelopmentinexceptionalcircumstances(pp.165-176). London: Churchill Livingston.

Rondal, J.A. (1988b). Language development in Down's syndrome: a lifespan perspective. International Journal of Behavioural Development, 11, 21-36.

Rondal, J.A. (1994). Exceptional cases of language development in mental retardation. The relative autonomy of language as a cognitive system. En H. Tager-Flusberg (Ed.), Constraints on language acquisition: studies of atypical children. Hillsdale, NJ: Erlbaum.

Rondal, J., Perera, J., Nadel, L. (2000). Síndrome de Down: Revisión de los últimos conocimientos. Madrid, Espasa.

Roizen, N. J. \& Patterson, D. (2003). Down's syndrome. Lancet, 361, 1281-1289.

Shapiro, B. L. (1983). Down syndrome: A disruption of homeostasis. American Journal of Medical Genetics, 14, 241-269.

Simoni, G., Brambati, B., Danesino, C., Rossella, F., Terzoli, G. L., Ferrari, M. et al. (1983), Efficient direct chromosome analysis and enzyme determinations from chorionic villi samples in the first trimester of pregnancy. Human Genetics, 63, 349-357.

Steele, M. W. \& Bregg, W. R. (1966), Chromosome analysis of human amniotic fluid cells. Lancet, 1, 183-385.

Stathopulu, E., Mackie Ogilvie, C. \& Flinter, F. A. (2003), Terminal deletion of chromosome $5 p$ in a patient with phenotypical features of Lujan-Fryns syndrome. American Journal of Medical Genetisc, 119:363-6.

Strome, S. E. \& Strome, M. (1992), Down syndrome: An otolaryngologic perspective. Journal of Otolaryngology, 21, 394-397.

Tsao, C. Y., Wenger, G. \& Bartholomew, D. W. (2005), Cri du chat syndrome and complex karyotype in a patient with infantile spasms, hypsarrhythmia, nonketotic hyperglycinemia and heterotopia. American Journal of Medical Genetiscs, 134A:198-201. 
Tyrrell, J. Cosgrave, M., McCarron, M., McPherson, J.Calvert, J., Kelly, A. et al. (2001). Dementia in people with Down's syndrome. International Journal of Geriatric Psychiatry, 16, 1168-1174.

Warburton , D., Dallaire, L., Thangavelu, M., Ross, L. Levin, B. \& Kline, J. (2004). Trisomy recurrence: A reconsideration base on North America data. American Journal of Human Genetics, 131, 3-7.

Wilkins, L., Brown, J., Nance, W. \& Wolf, B. (1982). Clinical heterogeneity in 86 home-reared children with the Cridu-chat syndrome. Journal of Pediatrics, 102, 528-533.

Wilkins, L., Brown, J. \& Wolf, B. (1980). Psychomotor development in 65 home-reared children with Cri-du-chat syndrome. Journal of Pediatrics, 97, 401-405.
Wishart, J. G. \& Johnston, F. H. (1990). The effects of experience on attribution of a stereotyped personality to children wit Down's syndrome. Journal of Mental Deficiency Research, 34, 409-420.

Wisniewski, K. E., Wisniewski, H. M.\& Wen, G. Y. (1985). Occurrence of neuropathological changes and dementia of Alzheimer's disease in Down's syndrome. Annals of Neurology, 17, 278-282.

Wright, I., Lewis, V. \& Collis, G. M. (2006). Imitation and representational development in young children with Down syndrome. British Journal of Developmental Psychology, 24, 429-450. 